\title{
Bolus vs. continuous feeding: effects on splanchnic and cerebral tissue oxygenation in healthy preterm infants
}

\author{
Luigi Corvaglia' ', Silvia Martini', Barbara Battistini', Paola Rucci' ${ }^{2}$, Arianna Aceti' and Giacomo Faldella'
}

\begin{abstract}
BACKGROUND: Intermittent and continuous tube feeding modes are commonly used to feed preterm infants. However, there is no clear evidence regarding which method is better tolerated. We investigated the differences between bolus and continuous feeding in terms of cerebral and splanchnic oxygenation in healthy preterm infants.
\end{abstract}

METHODS: Thirty preterm infants underwent a simultaneous 6-h near-infrared-spectroscopy monitoring of cerebral and splanchnic oxygenation, during which they were fed twice through an orogastric tube: one meal was given as a 10-min bolus, and the other was given continuously over a period of $3 \mathrm{~h}$. Oxygenation trends over time were evaluated and compared between bolus and continuous feeding modes.

RESULTS: Cerebral oxygenation did not change over time and did not differ between the two feeding techniques. Splanchnic oxygenation changed significantly over time and differed between the two feeding techniques, with a significant increase after bolus feeding and a remarkable reduction during continuous feeding.

CONCLUSION: Bolus and continuous feeding modes influence splanchnic oxygenation in healthy preterm infants differently. Further studies are needed to investigate possible underlying mechanisms and potential effects on feeding tolerance.

N eonatal care has substantially improved over the past decades, leading to a significant increase in the survival rate of very preterm infants (1). Enteral nutrition is essential for the development of the gastrointestinal tract in the preterm population because it improves intestinal motor activity (2), enhances the release of gastrointestinal hormones with trophic effects (3), and ameliorates feeding tolerance (4). However, very preterm infants are unable to coordinate sucking, swallowing, and breathing (5); thus, they are usually fed via an intragastric tube, continuously or through intermittent boluses (6). Differences between continuous and bolus tube feeding in terms of weight gain, days to full enteral feeding achievement, feeding tolerance, and effect on necrotizing enterocolitis have been largely investigated. Currently, however, there is no clear evidence regarding the method that is better tolerated (7).
The effect of the two feeding techniques on cerebral and splanchnic oxygenation has not been extensively investigated. Regional tissue oxygenation results from a dynamic balance between oxygen supply and consumption; thus, a reduction in this parameter might reflect an impairment of systemic oxygen saturation (e.g., due to the occurrence of apneic episodes (8)), a decreased cardiac output leading to a reduction of peripheral perfusion (9), a hemodynamic variation within the examined tissue (e.g., regional vasoconstriction/vasodilatation), or a higher oxygen consumption required to satisfy the metabolic demand (10).

Near-infrared spectroscopy (NIRS) is a noninvasive technique based on the near-infrared light absorption spectra of cytochromes, which provides a continuous monitoring of regional tissue oxygenation by measuring the levels of oxygenated and deoxygenated hemoglobin within a specific vascular bed (11). Since the mid-1980s (12), NIRS has been increasingly used in neonatal intensive care units to assess regional blood flow and tissue oxygen extraction under both physiological and pathological conditions, especially in splanchnic and cerebral regions $(9,13,14)$. Moreover, the relation between splanchnic and cerebral oxygenation has been proposed as a marker for abnormal vascular processes affecting the gastrointestinal tract $(15,16)$.

Studies performed using Doppler ultrasound measurements in preterm infants have shown that, following bolus feeding, there is a significant vasodilatation in the mesenteric circulation $(17,18)$. In line with this finding, a concomitant increase in splanchnic oxygenation, evaluated by means of NIRS, has been described (19-21). On the other hand, data about hemodynamic and tissue oxygenation changes related to continuous feeding are scarce, as are data documenting differences between cerebral and splanchnic oxygenation related to continuous feeding.

For this reason, in this study, we aimed to describe changes in cerebral and splanchnic tissue oxygenation in healthy preterm infants after bolus feeding and during continuous feeding, highlighting the differences between the two feeding modalities.

\section{RESULTS}

Thirty healthy preterm infants were included in the study. The infants' characteristics are detailed in Table 1. There were no

${ }^{1}$ Department of Medical and Surgical Sciences, Neonatology and Neonatal Intensive Care Unit, S. Orsola-Malpighi Hospital, University of Bologna, Bologna, Italy; ${ }^{2}$ Department of Biomedical and Neuromotor Sciences, Unit of Hygiene and Biostatistics, University of Bologna, Bologna, Italy. Correspondence: Arianna Aceti (arianna.aceti2@unibo.it) 
differences in terms of infants' characteristics between those who started the examination with continuous feeding compared with those on bolus feeding. No adverse event was observed during the study period.

Overall, 2,133 5-min measurements were obtained, 1,061 following bolus feeding and 1,072 during continuous feeding. To rule out decreases in tissue oxygenation due to systemic oxygen desaturation, 5-min measurements simultaneous with hypoxic episodes (17/1,061 following bolus feeding and $25 / 1,072$ during continuous feeding) were excluded from the analysis.

Median (interquartile range (IQR)) cerebral tissue oxygenation index (TOI) value was 75.7 (69.4-91.1) after bolus feeding and 75.4 (67.9-91.4) during continuous feeding; median (IQR) splanchnic TOI value was 62.8 (49.6-77.4) after bolus feeding and 55.3 (40.4-69.8) during continuous feeding.

Cerebral TOI values following bolus administration and during continuous feeding did not differ at any time point, neither in relation to the baseline value nor between the two feeding techniques. As shown in Figure 1, the locally weighted scatter plot smoothing (LOWESS) curves (22) over time of 5 -min cerebral TOI values for bolus and continuous feeding

Table 1. Characteristics of the study population

\begin{tabular}{|c|c|}
\hline Characteristics & $(n=30)$ \\
\hline Birth weight, median + IQR (g) & $1,275(1,099-1,568)$ \\
\hline Gestational age, median + IQR (weeks) & $30.1(28.3-32.0)$ \\
\hline Male gender, $n(\%)$ & $14(47)$ \\
\hline \multicolumn{2}{|l|}{ Ethnicity, $n(\%)$} \\
\hline Caucasian & $27(90)$ \\
\hline Hispanic & $1(3)$ \\
\hline Black & $0(0)$ \\
\hline Asian & $2(7)$ \\
\hline \multicolumn{2}{|l|}{ Mode of delivery, $n(\%)$} \\
\hline Cesarean section & $22(73)$ \\
\hline Vaginal delivery & $8(27)$ \\
\hline \multicolumn{2}{|l|}{ Type of gestation, $n$ (\%) } \\
\hline Single & $24(80)$ \\
\hline Twin & $6(20)$ \\
\hline \multicolumn{2}{|l|}{ Feeding, $n(\%)$} \\
\hline Fortified human milk & $14(47)$ \\
\hline Standard preterm formula & $5(16)$ \\
\hline Mixed feeding & $11(37)$ \\
\hline $\begin{array}{l}\text { Diagnosis of anemia at any time before the } \\
\text { monitoring, } n(\%)\end{array}$ & $10(33)$ \\
\hline Need for red blood cell transfusion, $n$ (\%) & $9(90)$ \\
\hline \multicolumn{2}{|l|}{ At time of monitoring: } \\
\hline Postnatal age, median + IQR (d) & $16(12-36)$ \\
\hline Weight, median + IQR (g) & $1,620(1,492-1,747)$ \\
\hline Need for supplemental $\mathrm{O}_{2^{\prime}} n(\%)$ & $0(0)$ \\
\hline Patients developing NEC, $n$ (\%) & $0(0)$ \\
\hline Patients developing feeding intolerance, $n$ (\%) & $0(0)$ \\
\hline
\end{tabular}

modes were nearly overlapping, and, therefore, the time trend did not differ $\left(\chi^{2}=18.72 ; P=0.9922\right)$.

On the contrary, as shown in Figure 2, splanchnic TOI changed significantly over time in different ways during the bolus and continuous feeding modes: specifically, the mixed model showed that both the time trend and the estimated mean splanchnic TOI differed significantly between the two types of feeding $\left(\chi^{2}=161.35 ; P<0.0001\right.$ and $\chi^{2}=173.28 ; P<$ 0.0001 , respectively).

Compared with baseline, splanchnic TOI values recorded after bolus feeding significantly increased from $1 \mathrm{~h}$ and $10 \mathrm{~min}$ to $2 \mathrm{~h}$ and $30 \mathrm{~min}$ from meal administration. Conversely, when compared with baseline, splanchnic TOI values detected during continuous feeding exhibited a significant reduction from $1 \mathrm{~h}$ and $35 \mathrm{~min}$ to almost the end of the procedure. The difference between the two techniques in terms of splanchnic oxygenation was significant from $1 \mathrm{~h}$ and $15 \mathrm{~min}$, as shown in (Figure 2).

No difference in terms of cerebral and splanchnic TOI values and feeding tolerance was found when comparing infants exclusively breastfed, formula fed, and receiving mixed feeding, either during continuous or after bolus modes of feeding.

\section{DISCUSSION}

The results of this study indicate that bolus and continuous feeding modes are associated with different types of changes of splanchnic oxygenation in healthy preterm infants.

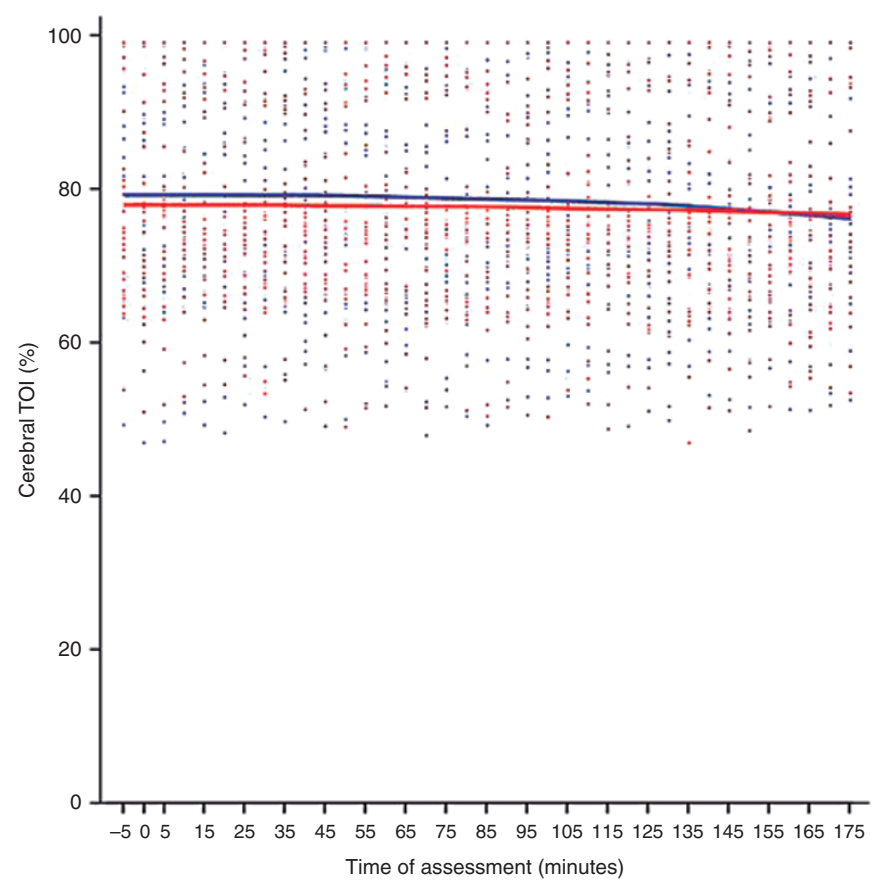

Figure 1. Scatter plot of cerebral oxygenation vs. time. Blue line and dots indicate bolus feeding. Red line and dots indicate continuous feeding. Dots represent the individual repeated measures of cerebral tissue oxygenation index (TOI) after bolus and during continuous feeding modes. Lines represent the estimated median cerebral TOI after bolus and during continuous feeding using locally weighted scatter plot smoothing fit. Time of assessment (minutes) is reported on the $\mathrm{x}$ axis; $0=$ start of feeding administration. 
Similarly to previous studies $(19,23,24)$, brain tissue oxygenation remained stable following bolus feeding and during continuous feeding. This is probably due to the physiological mechanism of cerebral autoregulation, which keeps the

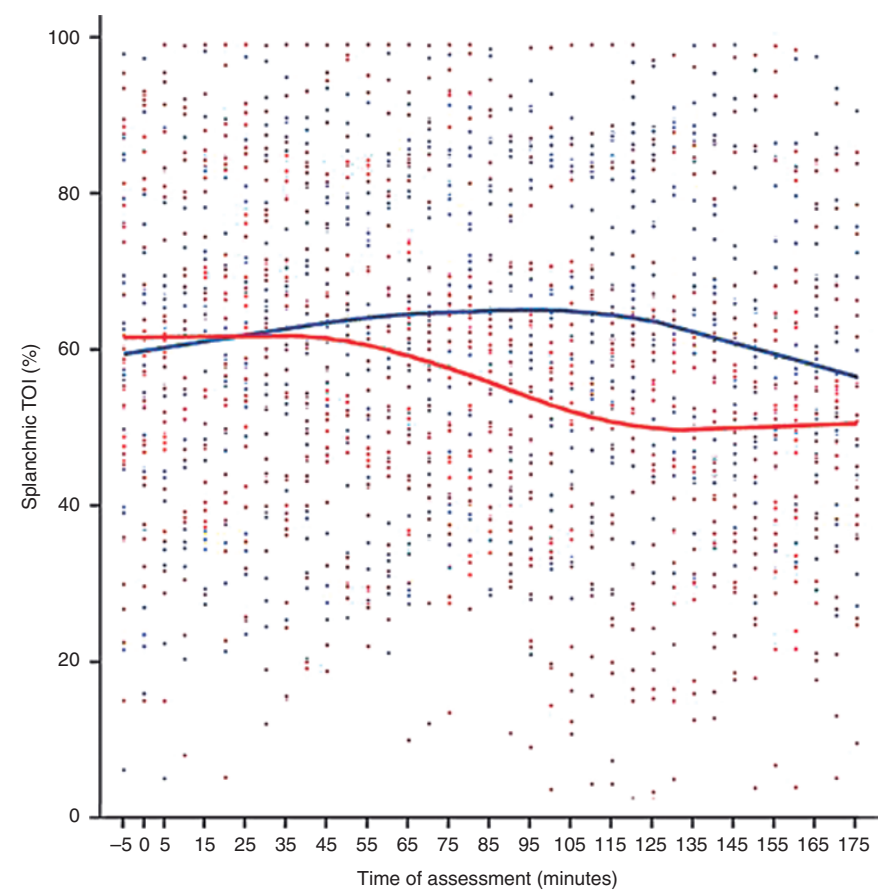

Figure 2. Scatter plot of splanchnic oxygenation vs. time. Blue line and dots indicate bolus feeding. Red line and dots indicate continuous feeding. Dots represent the individual repeated measures of splanchnic tissue oxygenation index (TOI) after bolus and during continuous feeding modes. Lines represent the estimated median splanchnic TOI after bolus and during continuous feeding using locally weighted scatter plot smoothing fit. Time of assessment (minutes) is reported on the $x$ axis; $0=$ start of feeding administration. levels of cerebral blood flow and of consequent oxygen delivery almost constant (25).

Interestingly, the two techniques differed significantly in terms of splanchnic oxygenation after a period of $1 \mathrm{~h}$ and $15 \mathrm{~min}$ from the beginning of feeding administration.

Previous studies that used Doppler ultrasound to analyze changes in splanchnic hemodynamics following bolus feeding in preterm infants documented a significant increase in mesenteric blood flow velocity after bolus administration $(17,18,20)$. Similarly, a significant increase in splanchnic oxygenation values, detected by NIRS, has been documented $(19,21)$, consistently with the previously described hemodynamic changes (20). It has been suggested that these variations may be due to the cyclic release of gastrointestinal hormones, such as gastrin, enteroglucagon, and gastric inhibitory peptide, specifically promoted by intermittent bolus feeding (3).

Dave et al. (19) documented a significant increase in splanchnic TOI values in stable preterm infants $1 \mathrm{~h}$ after bolus feeding; similarly, Dani et al. (21) showed a relevant increase in splanchnic oxygenation as early as $30 \mathrm{~min}$ from bolus administration. Consistently with previous studies, our results confirm a postprandial increase in splanchnic oxygenation, occurring from $1 \mathrm{~h}$ and $10 \mathrm{~min}$ onward. The higher splanchnic TOI values observed after bolus administration could be attributed to a rapid increase in mesenteric blood flow, which has been described in previous studies also $(17,18,20)$; however, currently, potential beneficial effects in terms of feeding tolerance can be only hypothesized and need to be specifically examined in future research.

To the best of our knowledge, Dani et al. (21) were the first to evaluate the effect of continuous enteral feeding on mesenteric blood flow and splanchnic oxygenation. According to their preliminary results, no difference was found between splanchnic oxygenation values recorded $30 \mathrm{~min}$ before, at the end, and $30 \mathrm{~min}$ after continuous feeding. In that study, however, they

Table 2. Exclusion criteria

\begin{tabular}{|c|c|}
\hline Exclusion criteria & Definition \\
\hline Feeding intolerance & $\begin{array}{l}\text { Occurrence of emesis and/or gastric residuals }>50 \% \text { of the previous feeding and/or } \\
\text { abdominal distension and/or bloody stools }\end{array}$ \\
\hline Major congenital abnormalities & Congenital heart diseases, gastrointestinal abnormalities, and malformation syndromes \\
\hline Central nervous system disease & Intraventricular hemorrhage grade $\geq 2$, periventricular leukomalacia \\
\hline Hypoxic injury & Hypoxic-ischemic encephalopathy and ischemic acute renal failure \\
\hline Necrotizing enterocolitis & Any stage \\
\hline Sepsis or other infection & Clinical and/or culture-proven infection \\
\hline Preexisting skin problems & Any skin disease (systemic or at the site of sensor placement) \\
\hline Intrauterine growth restriction & Abnormal antenatal Doppler blood flow \\
\hline
\end{tabular}

CPAP, continuous positive airway pressure. 
provided no information on tissue oxygenation changes that might occur during the procedure. We performed continuous monitoring of splanchnic and cerebral oxygenation levels for the whole feeding period, outlining the trends over time of both parameters. Our analysis showed a significant decrease of splanchnic oxygenation occurring in the second half of continuous feeding. Moreover, during the final $10 \mathrm{~min}$ of continuous feeding, splanchnic TOI showed a slight trend toward increased levels; hence, in accordance with the results of Dani et al., at the end of the procedure, the difference with the baseline TOI values was not significant anymore.

Our preliminary findings suggest that splanchnic tissue oxygenation is differently affected by the two enteral feeding modalities. Reduced splanchnic oxygenation has been proposed to be a predictive marker of feeding intolerance, sepsis (26), splanchnic ischemia (15), and necrotizing enterocolitis (14) in preterm infants. Considering this evidence, the response of splanchnic tissue oxygenation to continuous feeding needs to be carefully evaluated in larger studies focusing specifically on potential consequences related to feeding tolerance.

\section{Conclusions}

Bolus and continuous feeding modes represent the most common feeding strategies adopted in neonatal intensive care units. According to our results, the two enteral feeding techniques have different effects on splanchnic tissue oxygenation. Bolus feeding is associated with an increase in splanchnic oxygenation, which might reflect an increase in blood flow in the mesenteric region. On the other hand, continuous feeding is associated with a significant decrease in splanchnic oxygenation.

Further studies are required to assess the underlying physiological mechanisms and to evaluate possible effects of this finding on enteral feeding tolerance.

\section{METHODS}

\section{Study Population}

This study was performed in the neonatal intensive care unit of Sant'Orsola-Malpighi Hospital in Bologna (Italy). Inclusion criteria were the following: gestational age $<33 \mathrm{wk}$, enteral intake $\geq 100 \mathrm{ml} / \mathrm{kg} / \mathrm{d}$ of fortified human milk or standard preterm formula, and need for tube feeding. Exclusion criteria are listed in Table 2. Infants with patent ductus arteriosus, diagnosed by echocardiography, were excluded from the study because the presence of patent ductus arteriosus has been recently shown to affect levels of both cerebral and splanchnic tissue oxygenation $(27,28)$. Similarly, smallfor-gestational-age and intrauterine growth-restricted infants were not included because reduced flow velocities in the mesenteric vessels $(29,30)$ and impaired hemodynamic response to feeding $(31)$ have been documented in small-for-gestational-age infants with prenatal hemodynamic disturbances.

Written informed consent to participate in the study was obtained from the parents/legal guardians of each infant. The study was conducted in conformity with the principles and regulations of the Helsinki Declaration, and the protocol was approved by the Ethical Committee of Sant'Orsola-Malpighi Hospital, Bologna, Italy. The statistical analysis was carried out on blinded data.

\section{Feeding}

Each infant underwent a 6-h monitoring, during which he/she was fed twice via an orogastric tube. One meal was given as a 10 -min bolus: milk was poured into a syringe attached to the feeding tube and allowed to drip in by gravity; the orogastric tube was removed after bolus administration. The other meal was administered continuously over a 3 -h period using a feeding pump; the orogastric tube remained in place for the whole feeding period. The sequence of feeding modalities was alternated in study participants.

\section{Near-Infrared Spectroscopy}

To evaluate regional tissue oxygenation, each infant underwent simultaneously a continuous noninvasive monitoring of cerebral and splanchnic oxygenation levels with a NIRO-200 oximeter (Hamamatsu Photonics, London, UK), which is part of the routine care of preterm infants admitted to our neonatal intensive care unit. One sensor was placed in the central region of the forehead and the other on the region below the umbilicus, which has been shown to best sample the mesenteric circulation (32). To minimize changes in tissue oxygenation due to body positioning, infants maintained the supine posture during the whole monitoring period.

The recording was started after at least $30 \mathrm{~min}$ of NIRS monitoring. Cerebral and splanchnic oxygenation levels were quantified as TOI values and were recorded every $5 \mathrm{~s}$. TOI values were then clustered into 5-min intervals, and the mean TOI value for each interval was considered for statistical analysis.

To evaluate differences between the two feeding methods, each mean TOI value detected following the bolus feed was compared with the corresponding mean TOI value measured during continuous feeding. Moreover, for each of the two feeding techniques, each TOI value was compared with the preprandial TOI value, relative to the 5 min preceding the feed.

\section{Polysomnography}

A simultaneous, noninvasive polysomnographic recording (System Plus; Micromed, Mogliano Veneto, Italy) was performed to assess the occurrence of hypoxic episodes, which were defined as spontaneous decreases in $\mathrm{SpO}_{2}$ (oxygen saturation) $<85 \%$. Peripheral oxygen saturation was recorded by means of a polysomnography pulse oximeter sensor placed on the infant's foot. NIRS and polysomnography were started simultaneously, and the maintenance of synchronization was checked at the end of each monitoring. The abdominal band used for polysomnography recording was placed over the umbilicus, and thus it did not interfere with the NIRS splanchnic sensor.

\section{Statistical Analysis}

A repeated-measures mixed model was used to compare splanchnic and cerebral TOI values over time between bolus and continuous feeding modes. Mixed-effect models are used to analyze longitudinal data when repeated measures are taken for each study participant, but the number of observations per subject is not the same because of missing data. These models are named mixed because they allow testing of fixed effects (such as the type of feeding, which has only two possible modalities) and random effects, related to repeated measures taken on the same individual. The advantage of mixed models over repeated-measures ANOVA is that the former do not require complete data at each time point. Changes over time within individuals were tested separately from differences in mean TOI values (across all time measurements) between bolus and continuous feeding modes. The trend over time of splanchnic and cerebral TOI values was also examined graphically using locally weighted least-squares scatter plot smoothing (22). This exploratory procedure determines the shape of the fitting curve based on the empirical data, instead of fitting a polynomial of suitable degree, as in classic regression procedures. The problem with polynomial smoothing is in fact that a few data points at the extremes of the scatter plot can strongly affect the fitted values. LOWESS regression is based on a smoothing procedure that pays greater attention to the local points, i.e., the smoothed value of $y$ corresponding to a data point $x$ is obtained on the basis of the data points around it within a band of a certain width. The graph obtained with this smoothing procedure is a fitting curve that connects the estimated medians of the TOI values at each time point. Wilcoxon signed-rank test was used to compare the paired TOI values for bolus and continuous feeding at specific time points and to compare the number of hypoxic episodes recorded after bolus feeding with those observed during continuous feeding. Significance level 
was set at $P=0.05$. IBM SPSS Statistics version 20.0 (SPSS, Chicago, IL) and Stata's (StataCorp LP, College Station, TX) xtmixed procedure were used for data analysis.

Disclosure: Authors have nothing to disclose in connection with this paper.

\section{REFERENCES}

1. Larroque B, Bréart G, Kaminski M, et al.; Epipage Study Group. Survival of very preterm infants: Epipage, a population based cohort study. Arch Dis Child Fetal Neonatal Ed 2004;89:F139-44.

2. Berseth CL, Nordyke C. Enteral nutrients promote postnatal maturation of intestinal motor activity in preterm infants. Am J Physiol 1993;264(6 Pt 1):G1046-51.

3. Aynsley-Green A, Adrian TE, Bloom SR. Feeding and the development of enteroinsular hormone secretion in the preterm infant: effects of continuous gastric infusions of human milk compared with intermittent boluses. Acta Paediatr Scand 1982;71:379-83.

4. Berseth CL. Effect of early feeding on maturation of the preterm infant's small intestine. J Pediatr 1992;120:947-53.

5. Mizuno K, Ueda A. The maturation and coordination of sucking, swallowing, and respiration in preterm infants. J Pediatr 2003;142:36-40.

6. Schanler RJ, Shulman RJ, Lau C, Smith EO, Heitkemper MM. Feeding strategies for premature infants: randomized trial of gastrointestinal priming and tube-feeding method. Pediatrics 1999;103:434-9.

7. Premji SS, Chessell L. Continuous nasogastric milk feeding versus intermittent bolus milk feeding for premature infants less than 1500 grams. Cochrane Database Syst Rev 2011:CD001819.

8. Yamamoto A, Yokoyama N, Yonetani M, Uetani Y, Nakamura H, Nakao H. Evaluation of change of cerebral circulation by $\mathrm{SpO} 2$ in preterm infants with apneic episodes using near infrared spectroscopy. Pediatr Int 2003;45:661-4.

9. Mittnacht AJ. Near infrared spectroscopy in children at high risk of low perfusion. Curr Opin Anaesthesiol 2010;23:342-7.

10. Ferrari M, Mottola L, Quaresima V. Principles, techniques, and limitations of near infrared spectroscopy. Can J Appl Physiol 2004;29:463-87.

11. Victor S, Weindling M. Near-infrared spectroscopy and its use for assessment of tissue perfusion in the neonate. In: Kleinman C, Seri I, eds. Neonatology Questions and Controversies: Hemodynamics and Cardiology. 2nd edn. Philadelphia, PA: Saunders/Elsevier, 2012:151-72.

12. Brazy JE, Lewis DV. Changes in cerebral blood volume and cytochrome aa3 during hypertensive peaks in preterm infants. J Pediatr 1986;108: 983-7.

13. Goff DA, Buckley EM, Durduran T, Wang J, Licht DJ. Noninvasive cerebral perfusion imaging in high-risk neonates. Semin Perinatol 2010;34:46-56.

14. Oh S, Young C, Gravenstein N, Islam S, Neu J. Monitoring technologies in the neonatal intensive care unit: implications for the detection of necrotizing enterocolitis. J Perinatol 2010;30:701-8.

15. Fortune PM, Wagstaff $M$, Petros AJ. Cerebro-splanchnic oxygenation ratio (CSOR) using near infrared spectroscopy may be able to predict splanchnic ischaemia in neonates. Intensive Care Med 2001;27:1401-7.

16. Gay AN, Lazar DA, Stoll B, et al. Near-infrared spectroscopy measurement of abdominal tissue oxygenation is a useful indicator of intestinal blood flow and necrotizing enterocolitis in premature piglets. J Pediatr Surg 2011;46:1034-40.

17. Lane AJ, Coombs RC, Evans DH, Levin RJ. Effect of feed interval and feed type on splanchnic haemodynamics. Arch Dis Child Fetal Neonatal Ed 1998;79:F49-53.

18. Gladman G, Sims DG, Chiswick ML. Gastrointestinal blood flow velocity after the first feed. Arch Dis Child 1991;66(1 Spec No):17-20.

19. Dave V, Brion LP, Campbell DE, Scheiner M, Raab C, Nafday SM. Splanchnic tissue oxygenation, but not brain tissue oxygenation, increases after feeds in stable preterm neonates tolerating full bolus orogastric feeding. J Perinatol 2009;29:213-8.

20. Gillam-Krakauer M, Cochran CM, Slaughter JC, et al. Correlation of abdominal $\mathrm{rSO} 2$ with superior mesenteric artery velocities in preterm infants. J Perinatol 2013;33:609-12.

21. Dani C, Pratesi S, Barp J, et al. Near-infrared spectroscopy measurements of splanchnic tissue oxygenation during continuous versus intermittent feeding method in preterm infants. J Pediatr Gastroenterol Nutr 2013;56:652-6.

22. Cleveland WS. Robust locally weighted regression and smoothing scatterplots. J Am Stat Ass 1979;74:829-36.

23. Nelle M, Hoecker C, Linderkamp O. Effects of bolus tube feeding on cerebral blood flow velocity in neonates. Arch Dis Child Fetal Neonatal Ed 1997;76:F54-6.

24. Teller J, Schwendener K, Wolf M, et al. Continuous monitoring of liver oxygenation with near infrared spectroscopy during nasogastric tube feeding in neonates. Schweiz Med Wochenschr 2000;130:652-6.

25. Panerai RB, Kelsall AW, Rennie JM, Evans DH. Cerebral autoregulation dynamics in premature newborns. Stroke 1995;26:74-80.

26. Cortez J, Gupta M, Amaram A, Pizzino J, Sawhney M, Sood BG. Noninvasive evaluation of splanchnic tissue oxygenation using near-infrared spectroscopy in preterm neonates. J Matern Fetal Neonatal Med 2011;24: $574-82$.

27. Lemmers PM, Toet MC, van Bel F. Impact of patent ductus arteriosus and subsequent therapy with indomethacin on cerebral oxygenation in preterm infants. Pediatrics 2008;121:142-7.

28. Petrova A, Bhatt M, Mehta R. Regional tissue oxygenation in preterm born infants in association with echocardiographically significant patent ductus arteriosus. J Perinatol 2011;31:460-4.

29. Martinussen M, Brubakk AM, Vik T, Yao AC. Relationship between intrauterine growth retardation and early postnatal superior mesenteric artery blood flow velocity. Biol Neonate 1997;71:22-30.

30. Kempley ST, Gamsu HR, Vyas S, Nicolaides K. Effects of intrauterine growth retardation on postnatal visceral and cerebral blood flow velocity. Arch Dis Child 1991;66(10 Spec No):1115-8.

31. Pezzati M, Dani C, Tronchin M, Filippi L, Rossi S, Rubaltelli FF. Prediction of early tolerance to enteral feeding by measurement of superior mesenteric artery blood flow velocity: appropriate- versus small-for-gestationalage preterm infants. Acta Paediatr 2004;93:797-802.

32. McNeill S, Gatenby JC, McElroy S, Engelhardt B. Normal cerebral, renal and abdominal regional oxygen saturations using near-infrared spectroscopy in preterm infants. J Perinatol 2011;31:51-7. 